\title{
The Role of Community Health Agents in Northeastern Brazil: An Analysis of Health Knowledge Transfer in the Community
}

Renu Edpuganti*, B.A.

* To whom correspondence should be addressed: Harvard Medical School, 25 Shattuck St., Boston, MA 02115 , USA

\section{ABSTRACT}

The objective of this study was to describe and evaluate the health education component of a community health program in Pacatuba, a municipality in the Brazilian state of Ceará. The study focuses on health-related knowledge, measured through questionnaire-guided interviews, in the community and among the health agents as an indicator of program success. The sample size consisted of 18 health agents and 44 households, of which 28 were urban and 16 were rural. Comparisons between urban and rural households and general trends in knowledge transfer were examined. These observations then formed the basis for recommendations to increase program success. Overall, white agents revealed high levels of health-related knowledge, the results from the community sample varied. The community displayed proficient knowledge with respect to oral rehydration therapy preparation and use, the role of vaccines, and the purpose of prenatal exams. However, there was less subject knowledge regarding the function of child weighing, the importance of breastfeeding, and the knowledge of several family planning methods. Some of the factors believed to influence knowledge transfer to the community include the quality of health agent supervision, the supply of the agents' materials, and health orientations in the community. The possibilities for dealing with these concerns are discussed and elements for further program enhancement in health knowledge transfer, such as organizational stabilization and creative health education methods, are considered.

\section{INTRODUCTION}

The state of Ceará is located in Brazil's northeast region and is among the poorest states in the country (Fig. 1) (1). Almost two-thirds of the population of 6.5 million live below the poverty line and many do not have access to adequate health services or health education (2). Despite this, the state has recently been able to dramatically reduce its infant mortality rate (IMR). In 1987, the IMR in Ceará was 137 per 1000 live births. By 1990 this rate had dropped to 93 per 1000, a drop of 32\%. This is an accomplishment that not even the richer states of southern Brazil could lay claim to during the same period. As a consequence, this prompted UNICEF to recognize Ceará as an example for the world (1). 
The "Agente de Saúde" (Health Agent) program, run under the auspices of the State Secretary of Health, is considered to have played a crucial role in this achievement (1). The program originally began in 1987 in the capital city of Fortaleza in order to provide employment to individuals affected by the drought of the same year. It rapidly expanded thereafter as a relief effort to 128 surrounding municipalities of Fortaleza. In this way, the government could simultaneously provide income for these individuals as well as improve the health of the region. To provide relief as quickly as possible, the health agents' training lasted only 15 days, after which time they would work between 6 to 12 months supplying information to their communities in three priority health areas: breastfeeding, immunization, and oral rehydration therapy (ORT). Once the danger of the drought had passed in 1988, many of the municipal programs were phased out. However, the government, realizing the value of such work, decided to restore and restructure the program through state treasury resources.

The project, which has employed 7300 health agents since February of 1994, is designed to help communities promote and improve their own health care (3). It has served as a model for other states in Brazil (1). It is organized so that agents do not work at an assigned health post. Instead, their workday is composed of home visits. Furthermore, applicants do not need to meet any educational requirements to be an agent. In addition, agents have one direct supervisor who also works full-time in the same community. The health agents of the current program serve in 182 municipalities of the state's interior and act as links between the formal health system and the community. Each agent works full-time and is directly responsible for 100 to 200 families. They make at least one monthly visit, providing the families with much needed health education.

Agents in Ceará, whose primary job requirement is that they live in the community they serve, are compensated for their work with one minimum monthly salary, which at the time of the study was approximately equal to US $\$ 70$. Agents are selected based on an initial written test and an oral interview. In rural areas, where there may be few literate individuals, only the oral interview is used. Both classroom teaching and field-based learning take place over three months and include training with physicians, nurses, dentists, therapists, and social workers. Upon completion and certification by the coordinator of the course, agents may begin their work (4). Agents then serve as educators by orienting families on several priority health areas including breastfeeding, prenatal care, immunization, and diarrheal illness.

The goal of the present study is (i) to evaluate the efficacy of the health education component of the program currently running in the Cearán municipality of Pacatuba and (ii) to provide suggestions for improving the program. Specifically, this will be accomplished by measuring the level of knowledge of both the health agents in Pacatuba and a sample of the community. This will hopefully allow us to determine the extent of knowledge transfer between the health agents and the community. Knowledge transfer in this study refers to the knowledge present among health agents that is passed on to the members of the community.

\section{Project Site}

The municipality of Pacatuba is located 32 kilometers from Ceará's capital of Fortaleza. It is divided into four districts, one of which is the government seat, or Sede. This study involved only the Sede district. The Sede is composed of both urban and rural zones and has 18 health agents who serve approximately 1900 families. Because of this combination of zones, there is extreme variety in the living conditions within the Sede.

The Center area can be considered the "richest" of the zones. Most homes are equipped with modern conveniences such as electricity, piped-in water, toilets, and refrigerators. Because of easier access to health services and health education, In this area, households are generally not visited by a health agent unless a serious epidemic such as cholera or meningitis threatens the whole community. Beyond the Sede's Center lie other urban zones where the families have few modern conveniences available to them. The combined family income is generally around US $\$ 70$ per month (at the time of the study) and health agents visit these areas at least once a month. The third area, the rural zones, is located farther from the Center and is considered the 
poorest of the three zones. The rural zones may be anywhere from 40 minutes to 3 hours by foot from the Center's health facilities. Because these areas are the farthest from health services and information, many agents make weekly or biweekly visits to these areas.

Pacatuba's Municipal Secretary of Health has been enforcing the collection of data by health agents since 1992. As a result, information on several health statistics in the community is available from the municipal health office. Between 1992 and March of 1994 (just prior to the initiation of our study), figures related to maternal and child health show that in 1992,38\% of infant deaths in Pacatuba were due to diarrhea-induced dehydration. This dropped to $20 \%$ in 1993 and to $7 \%$ in January to March of 1994. The proportion of pregnant women who underwent full prenatal exams varied from $80 \%$ in 1992 , to $65 \%$ in 1993 , and then to $83 \%$ in the first three months of 1994 . The number of children under one year of age who were fully vaccinated ranged between $78-88 \%$ during this same time period. Finally, the rates of breastfeeding for infants aged zero to four months were lower in comparison to the other two areas with only $31 \%$ of these infants exclusively breastfed in 1992, 41\% in 1993, and 37\% in January to March of 1994.

\section{METHODS}

The field study was carried out between April and May of 1994. Data regarding general program information were gathered through direct observations and informal interviews, while questionnaire-guided interviews administered to health agents and community residents were used to measure their respective health knowledge.

All 18 of the Sede's health agents were interviewed. Their questionnaire seeked to gather information concerning general demographics, worker productivity, and health-related knowledge. The topics were selected based on prior interviews with the local supervisors who had trained the agents, as well as with officials at the State Secretary of Health who described the subject matter in which agents should be cognizant. Topics addressed in the questionnaire included diarrhea and dehydration, ORT, breastfeeding, water treatment and sanitation, immunizations, pregnancy and prenatal care, and growth monitoring. Agent scores were correlated with age, education, and experience using regression analysis to examine the relationships between such variables and health knowledge scores.

To examine the extent of knowledge transfer, community members from 44 households were interviewed. Health agents were accompanied on their regular home visits and the households were selected during these visits. The time and manpower constraints for the field portion of this study limited the size of the sample to 44. Sixteen of the interviews were conducted in the rural zones and 28 were conducted in the urban zones. Since urban households were located closer to each other, more visits could be made to urban households in one day than to rural households.

The household questionnaire included basic questions pertaining to demographic information, health behaviors and practices, and health-related knowledge. More specifically, community members were questioned about the purpose and function of vaccines and whether they could name diseases the vaccines protected against. Other questions addressed the perceived causes of diarrhea, the danger of dehydration, the purpose and preparation of ORT, breastfeeding and infant formulas, family planning, and the purpose of prenatal exams. Rural and urban results were analyzed using chi-square tests and individual scores were compared with the level of education using regression analysis. All interviews were conducted by the author to avoid interpersonal biases.

\section{RESULTS}

\section{Health Agents}


Although there are no educational requirements for being a health agent, all had completed at least a fourth grade education. More specifically, 4 of the 18 agents had completed fourth grade, 5 had completed fifth grade, 4 had completed part of high school, and 5 had completed high school. The median age of the health agents was 31.5 years. Six of the 18 agents had four years experience (since the program's inception in 1990), 11 had been working as agents for three years, and only 1 agent had been working for less than three years. Each agent in Pacatuba was responsible for making home visits to an average of 110 families. Agents reported visiting an average of five to eight households per day. This figure seems high and self-reporting bias may play a role.

Table 1 illustrates the level of knowledge among the health agents. All 18 were able to identify several symptoms of dehydration such as dry lips and delay in skin retraction when pinched up. All worker could also identify the major causes of diarrhea, which include poor hygienic conditions, untreated water, inadequately prepared food, and the presence of parasites, bacteria, or viruses. Regarding the treatment of diarrhea, 17 of the $18(94 \%)$ knew the importance of not suspending alimentation. The use of ORT in packet form and, more recently, in homemade form was advocated by all agents in cases of diarrhea. The correct use of ORT was taught throughout the community. With respect to the correct function of ORT, 17 of the 18 agents (94\%) understood that ORT serves to replace water lost during diarrhea. However, 28\% assumed incorrectly that ORT immediately stopped diarrhea upon use.

The level of knowledge regarding the diseases preventable by routine immunization varied greatly from $89 \%$ for tuberculosis, to as low as $61 \%$ for pertussis. All but one of the agents understood the modes of transmission and symptoms of both neonatal and adult tetanus, as well as the necessity for administering tetanus vaccines to pregnant women. With respect to the proper schedule for administrating vaccines, BCG was the only one correctly reported by all agents. The five agents that missed the correct measles immunization schedule did so because they did not know that the booster is required after one year.

With respect to maternal and child health and family planning practices, the knowledge was high regarding the reasons for advocating breastfeeding and knowing that breastmilk should be the sole source of infant nutrition for at least the first four months of life. Advantages mentioned by the group included: the complete requirements of vitamins, proteins, and water supplied in breastmilk; the lower risk of infant diarrhea associated with breastfeeding; and the lower risk of infant illness resulting from the protective properties of breastmilk.

Similarly, with respect to family planning, all agents were able to name some methods of contraception. However, they were limited to naming the oral birth control pill, condoms, and tubal ligation. Only $39 \%$ of the agents reported knowledge of other methods such as the intrauterine device and spermicides.

In contrast, knowledge was low with respect to recognizing the danger signs during pregnancy (such as swelling, bleeding, and high blood pressure). While all agents were capable of accurately describing the importance of regularly weighing children under two years of age to monitor their growth, many cited the difficulty of implementing this practice due to the shortage of weighing scales.

As for sanitation and water treatment, all agents knew the importance of burying feces at an appropriate distance from the house in the absence of bathrooms, and the need for adding hypochlorite solution to kill water-borne pathogens. A large portion (72\%) also suggested other methods of water treatment such as adding Q-boa, a chemical found in local stores to sanitize water, or filtering and/or boiling water before consumption.

Regression analysis of the agent's age and of the number of years of experience did not show either of these two variables to be significant predictors of health knowledge $(p>.05)$. On the other hand, regression analysis of the agent's highest completed level of education revealed that education was a significant 
predictor $(p<.03)$ and accounted for $23 \%$ of the variance. Multiple regression of the three variables was not significant $(p>.05)$.

\section{Community}

Table 2 illustrates the overall level of knowledge measured within the community. The data were separated into rural and urban zones to compare differences, if any, between the two areas. In general, the level of health-related knowledge within the urban zones was higher than that found in the rural zones. Knowledge was significantly different regarding the treatment of diarrhea (i.e. the function of ORT $[p<.04]$ ). Similar trends, while not statistically significant, were also observed in the areas of infant nutrition, identifying the causes of diarrhea, and the identification of required immunization, where the level of understanding was higher in urban households compared to rural households.

With several topics, the level of knowledge was similar in both the urban and rural population. These included the high understanding of the purpose of vaccination, the use of ORT in response to diarrhea, and the importance of prenatal exams. Interestingly, the overall proportion of households not using ORT (30\%) corresponded with the percentage of agents incorrectly believing that ORT was used to stop diarrhea (28\%).

With respect to the overall level of knowledge within the communities, there was a considerable lack of understanding for most topics. The greatest deficiency was seen in the following topics: the causes of diarrhea, the purpose of ORT, the proper sources of infant nutrition, the usage of birth control, and the purpose of weighing children. In addition, few people in either the urban or rural groups knew what dehydration was (25\% urban, $13 \%$ rural), and only some were able to at least associate it with diarrhea (32\% urban, $50 \%$ rural).

Regarding the level of education measured within the community, the urban households had a significantly higher level of education $(p<.03)$. While half of the rural population had no formal education, $78 \%$ of the urban population had at least a first grade education and $18 \%$ had at least a fifth grade education. Interestingly, however, regression analysis of the highest level of education completed against individual health knowledge scores did not show education to be a significant predictor of health knowledge $(p>.05)$.

\section{DISCUSSION}

Community health worker programs have been implemented in Brazil and other developing countries as a method of delivering primary health care to underserved populations. Many of these programs have the common theme of training local members of the community so that they can provide basic health services and health education to their respective communities and act as extensions of the formal health system.

As our findings illustrate, health agents in Pacatuba had a good overall comprehension of health information. However, there were some areas where the level of knowledge among the health agents was relatively low. These included the correct schedule for measles vaccination, the correct identity of certain diseases preventable by vaccination, the correct function of ORT, alternate methods of birth control, and recognizing the complications of pregnancy.

Regarding the level of knowledge in the community, some members showed a relatively adequate level of knowledge in selected topics. However, in general, there was a great lack of overall understanding for most topics. The topics which showed substantial amounts of community knowledge relative to the other topics included the use of ORT in response to diarrhea, the importance of prenatal exams, and the importance of vaccines.

The high level of knowledge regarding the importance of prenatal exams may be related to the education provided by agents as well as to the greater access to prenatal services at the health post. (The municipal 
health post has one staff nurse who sets aside two days each week to provide prenatal exams for pregnant women in the community.) In a similar fashion, the high proportion of families understanding the general use of vaccines to prevent disease may be related, in part, to the agents' teaching, as well as to the requirement that each child have a vaccination card that is stamped at the health post when vaccines are administered. Agents ask to see these vaccination cards during home visits to have proof that the children are being immunized. This makes it easier to judge which families are carrying out proper health practices and which require more education and encouragement. In addition, it allows the families to observe directly the immunization schedule.

However, despite these promising findings, community knowledge was low in most topics addressed. The low proportion of families who knew that breastmilk is the best source of nutrition for infants may be due to weaknesses in efficiently transferring this knowledge. Many mothers work outside the home or in the field and find it difficult to provide the appropriate breastfeeding that an infant needs. Instead, they may be more willing to follow advice from family members or neighbors who suggest other sources of alimentation for the newborn infant. Furthermore, the advertising of infant formulas in local stores often makes these products seem more modern and therefore superior to breastfeeding, thus competing with the information that families receive from health agents.

Concerning family planning practices, only the birth control pill, tubal ligation, and less often, the condom were commonly used methods of contraception. As shown in Table 1, few agents themselves were capable of naming any other forms of contraceptive methods aside from these three, suggesting a direct relationship between agent and community knowledge. This close relationship was also observed in the use of ORT for managing diarrheal illness. The proportion of the community that did not use ORT paralleled the proportion of agents who thought that ORT always stopped diarrhea. If families were told that ORT would stop diarrhea, and when they tried ORT the diarrhea continued, they would doubt its effectiveness and be less inclined to use it.

An important difference existed between urban and rural households. Since urban areas had greater exposure to newspapers, radio, and TV, which feature health announcements regarding immunization, ORT, and diarrheal illness, they may have had better access to health information prior to the start of the program. This in turn could have affected the ability of the urban population to retain the information presented to them by the health agents.

The lack of a significant association between education level and community scores suggests that knowledge retention may not be entirely dependent on the amount of education one has completed. These initial data may be encouraging, considering the generally low education level of the target group. These preliminary findings also warrant further research to better understand those factors which have a significant role in the retention of health knowledge.

Overall community knowledge and general health statistics are lower than what the government had designed the program to establish, suggesting that the program has room for improvement. Previous studies evaluating the benefits of similar programs have demonstrated noticeable improvements in the overall knowledge, attitudes, and practices of the communities served by these programs (5-9). These studies claimed that several important factors were responsible for the effectiveness of these health programs. These include the quality of training, support, and supervision of health workers; the size of the target area served by health workers; the supplies for health workers; and the levels of community organization, collaboration with local health services, and political involvement and support (9-12). These various factors can greatly influence the transfer of knowledge from the health agents to the community. This transfer is important to promote preventive health behaviors and to increase program success (7).

One crucial obstacle hindering the efficiency in Pacatuba is the limited organizational structure of the 
program. In each community, the program coordinators have the responsibility of overseeing the health agents' work, organizing meetings and refresher courses, collating the agents' monthly productivity reports, and acting as a liaison with the governmental organization. A coordinator that is strictly responsible for the supervision of the agents has been difficult to achieve in Pacatuba, as well as in similar programs in other countries (13-15).

During the initial four years, the Pacatuba program has had five individuals take on the position of coordinator, only to leave shortly thereafter. Furthermore, it is not uncommon for a month or more to lapse before a new coordinator is hired, leaving the agents without any careful supervision (16). Only one coordinator over the past five years has kept the position for a substantial amount of time (two years) to keep the program running smoothly. Within the past year alone, three different coordinators have held the position.

This constant transition of coordinators is a clear obstacle that limits the success of the program. It becomes difficult for such a short-term leader to establish a solid agenda and to keep the program running smoothly (17). Progress and expansion of the program becomes hampered. As a result of this constant changeover, agents lose much of their guidance, and the knowledge flow to the community slows down.

Another important effect of the lack of organization is that agents in Pacatuba are unable to take part in regular refresher courses, which would ordinarily be organized by the coordinator. Such courses would be useful to introduce new concepts in the health field and to reinforce previous knowledge, which would help maintain the community's confidence in the health agents' capabilities. A recent study of a similar program reported that frequent meetings and regular refresher courses organized by supervising staff resulted in greater knowledge levels among the community health workers and in the community (6). The consequences of the lack of such courses are evident in the health agents' low knowledge of certain new health issues, such as the need for a measles booster (a procedure only recently added to the immunization schedule) and the reinforcement of previous issues such as the potential dangers during pregnancy. The level of knowledge among health agents may have been higher had this information been provided through refresher courses.

Although agents stated that their initial training had been sufficient, they also asserted that refresher courses would be greatly beneficial in order to strengthen the ideas in their minds. Specific areas that were suggested include vaccine application, treatment procedures, and family planning practices.

Furthermore, aside from reinforcing health information, refresher courses with the coordinator can also be used to further develop agents' problem-solving abilities (18). This can also be used as a forum to assess progress in the past months and to determine what actions (i.e., educational techniques, meetings) can be taken to improve health practices in the community (17).

It seems clear that an important area of the program that needs immediate attention is its structural and administrative stability. Pacatuba needs a coordinator that has a chance to establish a solid program design. Coordinators in the past have left due to a lack of recognizable responsibility and to a lack of financial compensation. Overcoming these kinds of obstacles is not easy, for it depends on the cooperation of both the municipal and state governments (19). However, such cooperation is absolutely necessary for the success of the program.

Another limitation to the program's success is the lack of sufficient materials and medicines available to the agents to carry out their work. These materials include first aid kits, hypochlorite solutions, ORT packets, and weighing scales. The lack of scales means that families must take their children to the health post to be weighed. Practically speaking, this can only be achieved during the time of immunization, as it is not feasible for many families to make a trip to the health post each month for weighing. One suggestion provided by an agent was to purchase several scales to be kept in the municipal office and signed out by agents as needed. 
Another measure for improving the program's success is to focus on changing the community's health orientation toward preventive behaviors rather than simply curative ones. Members of the community tended to make trips to the health posts only once an illness became symptomatic, and were less likely to do so for preventive measures unless urged by the agents (20). Because of this practice, the preventive behaviors advocated by agents become more difficult to teach and institute into practice. In order to effectively transmit this message, new methods must be devised to instill the importance of prevention in the minds of the community members.

More creative and low-cost methods for transferring knowledge to the community can be used to help families retain this information and to realize the importance of preventive medicine. For example, the simple use of dolls, figurines, pictorial cards, and other visual aids can facilitate the understanding of complex medical concepts (21). These visual aids can be used during home visits and community meetings to make such events more interesting and to increase comprehension. Role playing among members of the community can also be effective (21).

Furthermore, efforts to implement preventive health behaviors in children can be expanded by increasing the participation of agents within the local schools. The program already features an oral hygiene program where agents visit schools to ensure that students are using correct brushing techniques and to apply fluoride to the children's teeth. Such links with the schools can be expanded so that agents can organize periodic information sessions concerning important adolescent health topics that are otherwise not commonly addressed, such as sex education and contraception. For children who must work at home and do not attend school, agents can organize similar central meetings closer to these children's homes. Initiating healthy habits among children can, in turn, have a positive influence on their parents' health habits.

Agents have recently ventured into two other health areas that can be further improved: alternative nutrition and herbal/home remedies. Because adequate nutrition is difficult to achieve in the poor areas, alternative nutrition orientation would be useful to develop further. In addition, since herbal and home remedies are often an option to costly pharmaceutical medicines, agents who know of natural remedies teach them within their communities. Other agents work with local traditional healers in order to learn these treatments. Once the safety and efficacy of these methods are verified, a course could be developed in order to teach other community agents these traditional practices so that they can bring this information back to their respective communities.

Ceará's program in Pacatuba features several desirable characteristics that have helped its initial success. Agents are selected from the community with no educational requirements, they are financially compensated for their full-time work, and, theoretically, they should have a supervisor from the same community to provide direct contact and support. The program has played a role in several initial successes including the high level of knowledge regarding vaccines and vaccination coverage, the high numbers of pregnancies that are followed up with prenatal exams, and the high knowledge and use of ORT in cases of diarrheal disease.

The findings in this study suggest that improvements in the transfer of health knowledge to the community is dependent upon various inputs (e.g., quality supervision, visual aids, collaboration with local health services, and supply of materials) in addition to improvements in the health agents' teaching methods. The health agent program can utilize the information revealed in this study in order improve those areas most needed, especially the supervision of health agents, in order to help the health agent program in Pacatuba achieve further success. Agents in Pacatuba, through their home visits, have reached many people and have already begun to make a difference. Exposure to health information was present before the program was created and continues to exist. Unfortunately, these state-wide immunization campaigns, disease campaigns, and health announcements on the radio, on television, and in newspapers represent a sporadic, rather than a continuous source of information which may only reach a minority of people, specifically those that live in the Center of the Sede. As a result of the program now operating in Pacatuba, a community that previously had little 
contact with health information is now finding sources of adequate and reliable health education. Ensuring the sound infrastructure of the program and the continual development of the issues revealed through this study can certainly improve the health education component of the program, increase knowledge transfer, and ultimately help expand preventive health behaviors in the community.

\section{ACKNOWLEDGMENTS}

The author wishes to thank Dr. Maria "Auxiliadora" Sousa for her important guidance and support; the officials at the State Secretary of Health office; Ms. Glaucia Cavalcante, Coordinator of the Pacatuba Health Agents; and the entire staff of health agents in Pacatuba, especially Ms. Leila Ferrer e Silva and Ms. Elizabete de Sousa for their hospitality and assistance.

\section{REFERENCES}

1. United Nations Infant and Children's Fund. Diréito à Vida - Criança: Prioridade Estadual. Fortaleza, Ceará, Brazil: United Nations Infant and Children's Fund; 1993.

2. United Nations Infant and Children's Fund. Situacão Mundial da Infância. Fortaleza, Ceará, Brazil: United Nations Infant and Children's Fund; 1992.

3. Saúde Para Um Ceará Melhor: Agente de Saúde. Fortaleza, Ceará: Ceará State Government; 1991.

4. Zita D. Coordinator of health agent training, Pacatuba maternity health post. Personal interview; May 1994.

5. Melville B, Fidler T, Mehan D, Bernard E, Mullings J. Growth monitoring: the role of community health volunteers. Public Health. 109: 111-116; 1995.

6. Stock-Iwamoto C, Korte R. Primary health workers in northeast Brazil. Social Science and Medicine. 36(6): 775-782; 1993.

7. Afari EA, Nkrumah FK, Nakana T, Sakatoku H, Hori H, Binka F. Impact of primary health care on childhood and mortality in rural Ghana: the Gomoa experience. Central African Journal of Medicine. 41(5): $148-153 ; 1995$.

8. Kuhn L, Zwarenstein MF, Thomas GC, et al. Village health-workers and GOBI-FFF: an evaluation of a rural programme. South African Medical Journal. 77(5): 471-475; 1990.

9. Chaulagai CN. Urban community health volunteers. World Health Forum 14: 16-19; 1993.

10. Mathews C, van der Walt H, Barron P. A shotgun marriage - community health workers and government health services: qualitative evaluation of a community health worker project in khayelitsha. South African Medical Journal. 84(10): 659-663; 1994.

11. Mangelsdorf KR. The selection and training of primary health care workers in Ecuador: issues and alternatives for public policy. International Journal of Health Services. 18(3): 471-493; 1988.

12. Kuhn L, Zwarenstein M. Evaluation of a village health worker programme: the use of village health worker retained records. International Journal of Epidemiology. 19(3): 685-692; 1990.

13. Andriessen PP, Gotink MH, van der Endt RP. The village health worker project in Lesotho: an evaluation. Tropical Doctor. 20: 111-113; 1990. 
14. Walt G, Ross D, Gilson G, Owuor-Omondi L, Knudsen T. Community health workers in national programmes: the case of the family welfare educators in Botswana. Transactions of the Royal Society of Tropical Medicine and Hygiene 83: 49-55; 1989.

15. Bamisaiye A, Olukoya A, Ekunwe EO, Abosede OA. A village health worker programme in Nigeria. World Health Forum. 10: 386-392; 1989.

16. Silveira ME, technician, Agente de Saúde program, State Secretary of Health, Fortaleza. Personal interview; May 1994.

17. Wasik BH. Staffing issues for home visiting programs. The Future of Children. 3(3): 140-155; 1993.

18. Gagnon AJ. The training and integration of village health workers. Bulletin of the Pan American Health Organization. 25(2): 127-138; 1991.

19. Minayo MC, D'Elia JC, Svitone E. Programa Agentes de Saúde do Ceará: Estudo de Caso. Fortaleza, Ceará: United Nations Infant and Children's Fund; 1990.

20. Conceicao D, nurse supervisor, Agente de Saúde Program, Maternity Health Post. Personal interview; May 1994.

21. Manual para Agente de Saúde Escolar. Fundacão para Assistência ao Estudante; 1989.

\section{BIOGRAPHY}

Renu Edpuganti received her B.A. in Natural Sciences and Public Health in 1994 from Johns Hopkins University (Baltimore, Maryland). She is currently a first-year student at Harvard Medical School (Boston, Massachusetts). This project was undertaken at the School for International Training (Brattleboro, Vermont). Her research interests include community-based efforts to improve health care delivery, as well as alternative and traditional medicine. Her future specialty interest is pediatrics.

Copyright (C) 1995 by MJM 